\title{
ARMAND BOREL: A REMINISCENCE
}

\author{
NOLAN R. WALLACH*
}

Murakami, a classmate of my thesis advisor, Jun-Ichi Hano, accepted an invitation to lecture at Washington University just after I had finished the first draft of my Ph.D. thesis. The title of his talk was essentially the same as the proposed title for my thesis. Obviously, I was worried. As it turned out my worst fears were correct and except for some differences he lectured on my thesis. Although, at the time this seemed to be a disaster, in hindsight, I was very lucky. Murakami was a visitor to the Institute for Advanced Study (IAS) for that academic year and he was in communication with Armand Borel and Joe Wolf (who was also visiting the Institute). When Murakami returned he told Borel and Wolf about me and my work. Within two weeks I received a copy of the joint paper of Borel and Tits on reductive groups in the IHES journal. I still have and cherish that copy which is now about 40 years old. The other good outcome was an invitation from Wolf to come to Berkeley as a postdoc. This twist of fate gave me an indirect introduction to two of the best mathematicians working in a field that I was beginning to feel would be my niche in mathematics: Lie theory.

Berkeley at that time was THE place to go to have a position if your field was differential geometry. Since, at that time, I considered myself to be a differential geometer I jumped at Wolf's invitation. I spent the next 3 years at Berkeley as an instructor (you could only be an assistant professor if you had published your thesis and Murakami had done that). At the end of the three-year period I left Berkeley for Rutgers partially on the suggestion of Wolf since he would be spending a year there to decide whether he would accept their offer of a distinguished professorship. Wolf guided me to my first visit to the IAS and we were both regular members of the audience for Harish-Chandra's lectures on representations of (so called) p-adic groups (later to be written up by Van Dieck). These lectures were traumatic to two differential geometers. I can remember how puzzled I was when Harish-Chandra said "let $K$ be a compact open subgroup of $G$ ". Wolf pointed out Borel in the audience. He was already a distinguished, gray, unapproachable presence. Harish-Chandra was also at least as formidable to me.

Some time that fall, Hesse and Earl Taft invited my wife, Barbara, and me to one of their Princeton parties. Borel was there and standing by himself in a corner. I finally walked up to him and said "Professor Borel, I would like to introduce myself. My name is Nolan Wallach." I had prepared other things to say, but before I could say them he said (here I am paraphasing) "Ah, Wallach (pronounced Vallach with a German ch) your Theorem 8.3 is false." I immediately walked over to Barbara and told her that I didn't feel well and would like to leave. We returned home and I immediately checked the result that Borel had mentioned. It was false, but easily fixed. I fixed the theorem and I sent the revision to Borel a few days later. The next time I saw Borel, he and I were at the afternoon tea at Princeton University. I approached him and asked if he had received the latest version of the paper. He said he had and the result was now correct but Rallis had a simpler proof.

For the next few years I would see Borel at Harish-Chandra's lectures and other seminars in Princeton and at the IAS. He congratulated me on my paper in the Annals

\footnotetext{
* Department of Mathematics, University of California at San Diego, La Jolla, CA 92093, USA (nwallach@math.ucsd.edu).
} 
on homogeneous Riemannian manifolds with strictly positive curvature. He was still an awesome presence but now and then I could detect a smile when we spoke. I had the feeling that he actually might like me. In hindsight I can say that I was correct. In the end I felt that I could count the two foremost masters of Lie groups, Borel and Kostant as my friends. But I had a long apprenticeship before that would happen.

In the Spring of 1976, Borel called me to propose that we do a seminar at the IAS. He said (in his deep voice) "The time has come to do a seminar on continuous cohomology, arithmetic groups and representation theory." He said that he would like for the two of us to do the seminar sharing in the lecturing and in writing the lecture notes as a volume in the Princeton University Press Lecture Note series. The first thing that came to my mind was "Why me?" Of course, I agreed to do the joint seminar. During the year of the seminar (1976-7) I learned how a great man works. I finished the year as a mature mathematician. Our seminar was on Tuesdays after lunch (and Harish-Chandra's morning seminar). After our weekly seminar lecture Borel and I would talk about the mathematics that would be appearing in the later lectures. A great deal of it was either new or at least went beyond what was known at the time. On Tuesday evenings when I would return home from my Tuesday marathon I would have a full week of work to do in preparation for the next Tuesday. Barbara no doubt would dread my return on those days since it was always late and I was exhausted. During the first month of the seminar I was still referring to Borel as Professor Borel (I had never known him by any other name). One day he said, "referring to me as Professor Borel is pretentious. I'm not that much older than you are. Call me Armand." The first few times I used Armand to address him I felt awkward and impertinent. Now referring to him as anything but Armand seems strange.

The seminar had three goals. The first (and most important to Armand) was to lay the foundations of the relationship between relative Lie algebra and continuous cohomology theories with coefficients in a unitary representation and the cohomology of local systems over a compact quotient of a symmetric space. The second was to develop continuous cohomology in the p-adic case and then to combine the Archimedian and non-Archimedian cases into the s-arithmetic case. The third goal (and most important to me) was to prove a vanishing theorem for relative Lie algebra cohomology to the real rank for simple Lie groups over the reals with coefficients in a non-trivial unitary representation. During Armand's lectures I learned that he had shown that Matsushima's method of proving his vanishing theorem could be adapted to prove theorems about unitary representations. This idea of proving vanishing theorems for representations and then using the results to prove vanishing theorems for local systems was not new but Armand understood it (I think) better than anyone else. The idea was implicit in Matsushima's formula for cohomology of compact quotients of symmetric spaces, the work of Hotta and me on $(0, p)$-cohomology of locally Hermitian symmetric spaces and Casselman's work in the p-adic case. In the end Armand and I (and Zuckerman independently) fulfilled our goal and, perhaps, gave the first application of the Langlands classification of irreducible admissible representations of real reductive groups. In addition, we showed how our method of proof of the Langlands' quotient theorem could be used to prove the analogous theorem for p-adic groups (proved independently by Silberger). I have had the good fortune to do intensive work with extraordinary mathematicians and each has taught me something new about how to think about mathematics. Armand's lesson was of a different nature than the others. He taught me what it means to be a professional mathemati- 
cian. His strongest criticism of a piece of work was "this looks like it was done by an amateur".

After the seminar we had a set of lecture notes that we had copied and distributed. These notes were, with some editing and clarification, Armand's goal for the seminar. I had hoped for more: a coordinated whole in the form of an informal treatise. At first Armand was against this plan (realizing now how much additional work was necessary in the transition I would now be on Armand's side on this issue) but he eventually relented. Throughout the year 1977-8 (during which I was a professor at Brandeis) I regularly commuted to the IAS to work with Armand on the transition. We had rented our house in New Jersey for that academic year and the tenants could not leave before August. So our family spent the month of July in the IAS residences. Armand and I used that time to put the finishing touches on the manuscript. We would work non-stop from early (for me) in the morning to early evening. One Friday Barbara decided that our family should spend the next day together. I had several things that I had to get to Armand on Saturday (which was a regular work day for him). I decided to drop the work in his mailbox very early and get back to my family to spend the day. I went over to his building at about 6:00 AM and dropped the material in his box (there were no special locks on the doors as there are now) and began my escape. I heard my name (as only he said it) "Vallach!". There was Armand on his bicycle! We completed the notes that summer. They were then proofed at every level: First during the typing done at the IAS; then the editing of the proofs (at least twice); and then the edge proofs. The day that the book was ready to actually be printed Armand called me up with good news. That we could go through the manuscript one more time. I am still amazed that with all of that editing if you open the book to a random page you will find a typo.

After the publication of our book in the Winter of 1980, Armand and I would talk mathematics now and then and we maintained our friendship (as you shall see) but we never worked together that intensively again. In the Summer of 1977, Armand and Bill Casselman organized what has now come to be called the Corvalis Meeting. The idea of the meeting was to set the direction for the future work on the Langlands Program. I had recommended the meeting to all of my younger friends in mathematics (such as my former student Welleda Baldoni Silva, Tom Enright and David De George). I told them that it would be a landmark meeting which would set the direction of mathematics for the rest of the 20th century. In hindsight, it was a landmark meeting, but it did not set the direction for the work on the Langlands program. My feeling was that it was premature. I was asked to lecture on the representations of real groups and contribute to the first Corvalis volume (the second was the serious work on the Langlands philosophy). The stamp of Armand's will on the meeting was obvious. I (and the friends I had talked into coming to the meeting) felt like we were in boot camp. The meeting was on the campus of Oregon State University in Corvalis. In addition to the mathematics meeting there was a football camp and a cheerleaders camp. Larry Corwin and I decided that it would be a good idea to have a tee shirt for the mathematics group in the orange (perhaps because of Princeton, on this I can't remember and sadly Larry passed away in his youth). The tee shirts said Armand Borel Math Camp. Armand's tee shirt said in addition Coach and Bill Casselman's said assistant coach. Bill was furious. I guess he thought he should also be a full coach. Armand clearly enjoyed the joke. Gaby Borel, took a picture if the participants (I can't remember why I wasn't in the picture) in their tee shirts.

In the Summer of 1980 the daughter of the president of Romania organized a 
meeting in Constanza on Operator Theory and Representation Theory. One of the advertised participants was I.M.Gelfand. I accepted the invitation to participate in the meeting just so that I could meet Gelfand. Armand also participated. Gelfand arrived at the meeting a week late. I remember vividly the look of affection that Gelfand had in his eyes when he saw Armand at this meeting. The week in Constanza was another important transitional period for me since I was able to talk intensively with Gelfand. In addition to having insights to every aspect of mathematics Gelfand was also a very opinionated philosopher. Once we were talking about why some mathematicians (such as himself and to me at that time Borel) could continue to do top notch creative work into their old age while others are "burned out" in their 40's. His explanation was that to the former mathematics was still fun. It is interesting that Armand's older daughter said something similar about her father at the memorial afternoon in his honor in the Fall of 2003. (I am paraphrasing here.) "My father approached all things with the enthusiasm of a curious child".

At the International Congress of Mathematicians in Helsinki, 1978, Jacque Tits approached me about writing a book on the representation theory of real reductive groups for Springer Verlag. I had been thinking about such a project for several years and I indicated that I was interested. I told Tits that I would work on the manuscript at my own rate without signing any contracts until the book was essentially done. I talked to Armand about the project in about 1985 and he suggested that I publish with the Academic Press. To me the Academic Press green series was the place to publish for three reasons. The first was that Helgason's classic book was published in that series. The second was that the books never went out of print. (How things change!) The third was that Armand was one of the editors. During the time I was preparing my manuscript Academic Press went through a wrenching transition which included the loss of A. K. Peters who had been the guiding light of that great series. Armand was outraged but he agreed to stay on as an editor until my book was done. As an editor Armand was as scrupulous as he was as a writer.

For several years, beginning in 1983 Armand had joint positions with the IAS and the ETH in Zurich. During his time in Zurich he set up a seminar (now known as the Borel Seminar) which brought together mathematicians from all over Switzerland. The seminar met at the University of Bern which is strategically located in the train station. The participants would travel by train from the various universities in Switzerland to participate in the seminar. There are two classic lecture note volumes that came from these seminars: one on perverse sheaves and one on D-modules. Both contain a main lecture by Armand laying out the theory that would be explored in the other lectures. I visited him in Zurich during the summer of 1983. During that time his other visitors included Goresky, McPherson, Bernstein and Casselman. One curious outgrowth of his renewed relationship with Switzerland was that he became an American citizen during that time. I don't know the details of the reason for his having broken ties with Switzerland but I am sure that the main loss was to the development of Swiss mathematics.

In the winter of 1990 I left Rutgers to accept a position at the University of California, San Diego (UCSD). When, I arrived at UCSD, I found that they had a wonderful program for distinguished visitors. During my first quarter there the visitors were Hörmander, Karubi and Macdonald. There was also a program for shorter distinguished visits. I invited the Borels to visit during the next year. Armand came first by himself and Gaby two weeks later (as I remember). Armand did his swimming religiously every day early in the morning. My job in preparation for his 
arrival was to set up for his use of one of the UCSD swimming pools at about 6:00 in the morning. The only available method was for Armand to join the Masters swimming class. The deal that was struck was that they would keep a lap open so that Armand could do his swimming in the wee hours of the morning. That visit of Gaby and Armand cemented a bond between our two families. Barbara and I and the Borels had become good friends. Also, I began to understand the important role Gaby played in Armand's long creative career.

In addition to Armand's visits to UCSD, I saw Armand during my annual visits in the fall to the New York area. These visits were set up to give lectures or to participate in the FOCUS committee of the Center for Communications Research. But in addition they were for the purpose of visiting three elderly aunts who lived in Brooklyn and Manhattan. During the entire time I knew Armand, he and Gaby would spend some part of the summer in Switzerland. During this time Gaby would stay on after Armand left to care for her mother. I would get together with Armand and perhaps go to a restaurant for dinner. Our dinner conversations covered a gamut of subjects. Armand had no use or understanding of small talk. When he spoke you knew that he had seriously thought through everything that he said.

The purpose of the Borels' last visit to San Diego in 1998 was to complete the manuscript of the second edition of our joint book which was published by the AMS. Armand had the original text Xeroxed in a magnified form. The plan was to edit the original text as if it were galleys and to insert the new material in the pertinent places. The rule was that the references by chapter, section and subsection in the original edition should be correct in the second edition. Every part of the book was meticulously studied and every word debated. This book was, however, of a different nature than the first edition. The field had become a standard part of the subject and the novelty of the first edition was gone. The purpose of the second was to keep the reference available and to update it with at least part of the amazing research that had been done in the intervening 20 years. As in his previous visit Armand swam with the Masters class.

In 1999 Armand accepted a special position at Hong Kong University which involved a fixed one year stipend for him and money for visitors. Armand decided that he would divide his stipend into 3 pieces and do a three year program (Programme in the listing in Hong Kong) which was called the Programme in Lie Groups. I participated in the programme during its second year. My job was to lecture on the "real case" and prepare for Barbasch's lectures in the "p-adic case" (I fear that I left Dan with an insurmountable job). Barbara joined me in Hong Kong for the last two weeks of my visit. Gaby treated us almost like family and Armand seemed truly pleased that he could share that place that he loved with us. Armand had a genuine affection for Hong Kong and also mainland China. It was certainly appropriate that the last project that he planned was for a programme in China similar to the one he supervised in Hong Kong. Just before I left for my visit to Hong Kong Armand sent an email message to me saying that a major article on the cohomology of locally symmetric spaces with coefficients in local systems pointed out that a "standard text in the subject" contained an incorrect proof of the Hochschild-Serre spectral sequence for relative Lie algebra cohomology. That paper included an appendix with a proof of a very special case of the theorem stated in our first and second edition. The assertion of incorrectness was, in fact, correct. However, the "fix" was relatively simple. Armand and I spent a few days on it and put together a one page explanation of the changes necessary to give a corrected proof of exactly the statement in the book. I 
include that page as an appendix to this reminiscence.

The last time I saw Armand was at the European Union summer school on Lie groups in Luminy during the summer of 2001. The meeting was organized around three different topics with two main lecturers for each topic. My job was to give applications of representation theory and the trace formula to the geometry of locally symmetric spaces in preparation for Labesse lectures on the Selberg-Arthur trace formula. The second topic was on the theory of reductive symmetric spaces and there were lectures by Delorme and Schlichtkrull. The third was on compactifications of symmetric spaces by Borel and Lizhen Ji. The format was that the lectures in each topic would be divided between the assigned lecturers. The lectures by Borel and Ji involved their recent joint work on the unification of the various methods of compactification that permeated the literature. At that time I was an editor for the Birkhauser series Mathematics: Theory and Applications. I approached Borel and Ji to publish an expanded version of their lectures in the series. They agreed and I am proud to say that I am an editor of Armand's last work which will be published as a posthumous joint volume with Ji.

Although I have been living in California for 14 years, my main source of news is a religious reading of the New York Times every morning. One morning in August, I saw a familiar picture of Armand in the obituary page. I could hardly read the well written obituary. I kept thinking, how is this possible? He was so strong and vital just two years earlier. A few days later we received a letter from Gaby explaining what had happened. In October of 2003 the IAS had an afternoon and evening ceremony to celebrate Armand's distinguished career. The evening was capped by a recital of Armand's new love, Indian music.

One day during our visit to Hong Kong Armand, Gaby, Barbara and I went to the bird zoo. It was an amazing jungle like area enclosed by a meshed canopy. The variety of birds was amazing. At one point I turned to Armand and said "If there were truly reincarnation I would like to come back as a bird." Armand turned to me and said "I wouldn't. Birds can't do mathematics".

Appendix. This appendix is the erratum for our book Continuous Cohomology, Discrete Subgroups, and Representations of Reductive Groups, second edition Mathematical Surveys and Monographs, Volume 67, AMS, 1999. It is reproduced in exactly the form that we had agreed upon in Hong Kong. The references in the body are all to this book.

Jens Franke in an appendix to his paper "Harmonic Analysis on Weighted $\mathrm{L}_{2}$ Spaces", Ann. scient. Éc. Norm. Sup., 31(1998), 181-279 points out that the proof of Theorem 1.6.5 contains an error based on Lemma I.6.4 which is false in its full generality. ${ }^{1}$ The lemma as stated is correct in the special case when $H=L$. This is the starting point for Franke's proof of the special case of the theorem that that is pertinent to his paper. This addendum describes how one can make an almost trivial modification of Lemma I.6.4 and of the proof of Theorem I.6.5 leaving the statement unchanged. The following discussion is an explanation of the necessary changes.

In the last sentence of the statement of Lemma I.6.4 replace injective by acyclic. At the end of the second to last sentence of the proof (line 1 of page 21 ) replace "for

\footnotetext{
${ }^{1}$ In fact, Franke's comment is not that specific. It refers to an error in "Some textbooks ..." based on an incorrect assertion about restrictions of injective modules. We have interpreted his statement in this way.
} 
some $U^{\prime} \in \mathcal{C}_{\mathfrak{n}, \mathfrak{k}_{1}, H}$." with:

where $U^{\prime}=\operatorname{Hom}_{S_{2}}(M, U)_{(H)}$ (see the proof of Lemma 8.6).

After the last sentence of the proof add the following material:

That being said, to complete the proof we need only show that if $U$ is a $(\mathfrak{k}, L)$ module then

$$
W=\operatorname{Hom}_{S_{1}}\left(U(\mathfrak{n}), U^{\prime}\right)_{(L)},
$$

with $U^{\prime}=\operatorname{Hom}_{S_{2}}(M, U)_{(L)}$, is acyclic in $\mathcal{C}_{\mathfrak{n}, \mathfrak{k} \cap \mathfrak{n}, H}$. If $\gamma \in \widehat{L}$ and if $Z$ is an $L$-module then $Z_{\gamma}$ will denote the $\gamma$ isotypic component of $Z$ relative to the action of $L$,as usual. We put $V=\operatorname{Hom}_{S_{1}}\left(U(\mathfrak{n}), U^{\prime}\right)_{(H)}$. Set $\widehat{L}_{1}$ equal to the subset of $\widehat{L}$ consisting of those elements containing the trivial one dimensional $H$-module as an $H$-submodule. Let $C^{\cdot}=\operatorname{Hom}_{H}\left(\bigwedge^{\cdot}(\mathfrak{n} / \mathfrak{k} \cap \mathfrak{n}), W\right)$. Then we must show that $H^{i}\left(C^{\cdot}\right)=0$ for $i>0$. Now

$$
\left.\operatorname{Hom}_{H}(\bigwedge(\mathfrak{n} / \mathfrak{k} \cap \mathfrak{n}), V)=\coprod_{\gamma \in \widehat{L}_{1}}\left(\operatorname{Hom}_{\mathbb{C}} \bigwedge \cdot(\mathfrak{n} / \mathfrak{k} \cap \mathfrak{n}), V\right)_{\gamma}\right)^{H} .
$$

Since $\mathfrak{n}$ is a finite dimensional $L$-module, it is easily seen that

$$
\operatorname{Hom}_{\mathbb{C}}(\bigwedge(\mathfrak{n} / \mathfrak{k} \cap \mathfrak{n}), V)_{\gamma}=\operatorname{Hom}_{\mathbb{C}}\left(\bigwedge^{\cdot}(\mathfrak{n} / \mathfrak{k} \cap \mathfrak{n}), W\right)_{\gamma}
$$

Now suppose that $i>0$ and $\omega \in C^{i}$ with $d \omega=0$. Then since $V$ is injective in $\mathcal{C}_{\mathfrak{n}, \mathfrak{k} \cap \mathfrak{n}, H}$, there exists $\eta \in \operatorname{Hom}_{H}\left(\bigwedge^{i-1}(\mathfrak{n} / \mathfrak{k} \cap \mathfrak{n}), V\right)$ with $d \eta=\omega$. A priori, $\eta$ is a possibly infinite sum of elements $\eta_{\gamma}$ with $\left.\eta_{\gamma} \in \operatorname{Hom}_{\mathbb{C}}\left(\bigwedge^{i-1}(\mathfrak{n} / \mathfrak{k} \cap \mathfrak{n}), W\right)_{\gamma}\right)^{H}$. However, if we set $\mu=\sum_{\gamma, \omega_{\gamma} \neq 0} \eta_{\gamma}$ then $\mu \in C^{i-1}$ and $d \mu=\omega$. This completes the proof of Lemma I.6.4 (revised).

In the proof of Theorem I.6.5 make the following changes. Replace the word "injective" and "injective, hence acyclic" with the word "acyclic". 
